\title{
On comparing zero-alpha tests across multifactor asset pricing models
}

\author{
Lieven De Moor* Geert Dhaene ${ }^{\dagger} \quad$ Piet Sercu $^{\ddagger}$
}

July 23, 2015

\begin{abstract}
Evaluating competing multifactor asset pricing models involves comparing the statistical significance of their mean pricing errors (alphas). Unfortunately, this comparison favors imprecisely estimated models because $p$-values tend to be higher in more noisy models. To avoid false impressions of relative success at tests for zero mean pricing errors, we develop a notion of comparative $p$-values and suggest comparing these instead of the raw $p$-values. This comparison gives more precisely estimated models a fairer chance or, equivalently, quantifies how much easier it is for imprecisely estimated models, by comparison, to pass the test.
\end{abstract}

Keywords: asset pricing, pricing errors, model comparison, multifactor models

*Faculty of Economic and Social Sciences and Solvay Business School, Vrije Universiteit Brussel, Belgium; lieven.de.moor@vub.ac.be.

${ }^{\dagger}$ Department of Economics, KU Leuven, Belgium; geert.dhaene@kuleuven.be.

†Leuven School of Business and Economics, KU Leuven, Belgium; piet.sercu@kuleuven.be. 


\section{Introduction}

To be deemed successful, a multifactor asset pricing model should meet two requirements. First, it should explain the expected excess returns better than alternative models that use different factors. Measures for this are the $R^{2}$ and significance tests for the factor exposures (for time-series regressions) or for the estimated prices of covariance risks (for cross-sectional regressions). Second, the covariance risks should explain all of the expected excess returns. This means not just that additional factors should be insignificant but also that, in regressions of excess returns on the factors, there should be no role for intercepts (the alphas). The latter condition can be tested by means of significance tests of the estimated alphas, separately or jointly. ${ }^{1}$ This paper focuses on zero-alpha tests and particularly on the issue of unequal power of the competing regressions.

In Fama and French (2012), the term "power" occurs 21 times and is concentrated in the discussions of the test results and the comparison across models. They distinguish two problem situations, those with "too much" and those with "too little" power. The first arises when a model's $R^{2}$ is comparatively high and the power is, therefore, probably adequate. The null hypothesis of zero alphas is then sometimes rejected even when the pricing errors are small. Second, it may occur that "the regressions fit less tightly and power is a problem" (Fama and French, 2012, p. 458) because then the zero-alpha null hypothesis is easily accepted even when the pricing errors are relatively large.

Thus, the twin criteria of a good fit and zero alphas often contradict each other, which has implications for the comparison of competing models. In particular, comparing $p$-values of the zero-alpha null across models is contentious because the precision of the estimated alphas differs across models. Similarly, in a GMM framework, comparing Hansen's (1982) $J$ statistics across alternative asset pricing models is controversial because it favors more noisy models (Jagannathan and Wang, 1996; Hansen and Jagannathan, 1997). Jagannathan and Wang (1996, p. 18) argue that "if a model contains

\footnotetext{
${ }^{1}$ There is a very large body of literature on (one- and multifactor) asset pricing models and testing. See, e.g., Jensen (1968), Black, Jensen and Scholes (1972), Fama and MacBeth (1973), Basu (1977), Banz (1981), Jobson and Korkie (1982), Gibbons (1982), Stambaugh (1982), Gibbons, Ross and Shanken (1989), Kandel and Stambaugh (1989), MacKinlay and Richardson (1991), Shanken (1992), Fama and French (1992, 1993, 1996, 2012, 2015), Jegadeesh and Titman (1993), Jagannathan and Wang (1996), Hansen and Jagannathan (1997), Carhart (1997), Pastor and Stambaugh (2003), Liu (2006), Ray, Savin and Tiwari (2009), Lewellen, Nagel and Shanken (2010), Asness, Moskowitz and Pedersen (2013), and Beaulieu, Dufour and Khalaf (2013).
} 
'more noise,' [...] then the value of the quadratic form will be smaller. In this case, it would be misleading to conclude that the "more noisy' the model, the better it performs." In the same vein, Cochrane (2005, p. 216) notes that "it has proved nearly irresistible for authors to claim success for a new model over previous ones by noting improved $J_{T}$ statistics, despite different weighting matrices, different moments, and sometimes much larger pricing errors." Again, the underlying problem is the unequal precision of the competing model estimates. In short, if a new model has "too little power" (i.e., its alpha estimates have greater standard errors than those in competing models) and rejects the zero-alpha null less often, one may well wonder about the extent to which this reflects poor precision rather than a genuine improvement. Likewise, "too much power" is a potential issue when the new model has more tightly estimated alphas and rejects the zero-alpha null more often.

In this paper, we compare zero-alpha $p$-values across models in a way that equalizes the precision of the estimated alphas. Our model comparisons are pairwise and we treat the models, new and existing, symmetrically. For a given pair of models and a singlealpha comparison, we revise the $p$-value of the model with the highest precision of the estimated alpha. Specifically, we ask what the $p$-value would have been had the precision been as poor as in the less-precise model. This means that we add to the estimated alpha of the more precise model a zero-mean random term with a standard deviation chosen such that the precision of the estimated alphas of the two models become equal. Each possible value of this random term implies a corresponding $p$-value of the zero-alpha null, and integrating this $p$-value over its distribution gives the comparative $p$-value, as we call it, of the more precise model. For the less-precise model, the comparative $p$-value is the same as the unmodified $p$-value, so that the two models can then be compared in terms of that metric. The comparison will reveal whether any success at the zeroalpha test of the lower-precision model relative to the more precise model is primarily due to its low precision rather than to a genuine improvement. For joint zero-alpha tests, the comparative $p$-values are defined similarly: for each model, we augment the estimated vector of alphas with a zero-mean random vector to equalize the precision of the model estimates and integrate the $p$-value derived from the ensuing Wald statistic over its distribution.

We emphasize that we make no claim whatsoever that the comparative $p$-values, taken individually, are any more valid than the original ones. Clearly, they are not: low 
precision cannot be conjured away. Our purpose is just to improve the way in which models with different precisions are compared and thus to avoid false impressions of relative success at zero-alpha tests when, in fact, the success is due to comparatively low precision. If we find, for instance, that a precisely estimated model is rejected given its low $p$-value but would be accepted in the light of its comparative $p$-value, we do not at all claim that the model's pricing errors are statistically insignificant. Rather, we note that, if they had been estimated as imprecisely as those in the imprecise model, we would not have worried about them. That conclusion qualifies the results for the imprecise model, not those for the precise one.

In Section 2, we review the link between $R^{2}$ and the power of zero-intercept tests. In Section 3, we introduce the comparative $p$-values and discusses their main properties. In Section 4, we give an illustration relating to the comparison between a liquidityaugmented CAPM and the Fama-French three-factor model. Section 5 concludes.

\section{Sources of power}

Fama and French (2012) treat $R^{2}$ and power almost as synonyms, but this misses a part of the picture that is relevant for our purposes. In a simple regression, it is true that, given the slope estimate, there is a one-to-one link between $R^{2}$ and the $t$-statistic for the slope, the first aspect of a CAPM test. But the relation with the second aspect, the $t$-test on the intercept, is more complicated. For illustrative purposes, consider a generic regression $Y=\alpha+\beta X+\varepsilon$ with, initially, one regressor and the classic OLS assumptions. We leave unspecified whether we consider a time-series or a cross-sectional test of $H_{0}: \alpha=0$.

Using standard notation, the OLS intercept estimate is $\widehat{\alpha}=\bar{Y}-\widehat{\beta} \bar{X}$, telling us that the variance of $\widehat{\beta}$ affects the variance of $\widehat{\alpha}$ unless $\bar{X}$ equals zero. In a successful CAPM time-series regression, however, $\bar{X}$ is the risk premium, so one cannot count on a zero $\bar{X}$ to eliminate the effect of imprecise betas. Likewise, in a cross-section test, $\bar{X}$ is an average covariance or an average beta, which is usually nonzero. The link between the variances of $\widehat{\alpha}$ and $\widehat{\beta}$ is

$$
\begin{aligned}
\operatorname{var}(\widehat{\alpha} \mid X) & =\operatorname{var}(\bar{Y} \mid X)+\operatorname{var}(\widehat{\beta} \mid X) \bar{X}^{2} \\
& =\frac{1}{n}\left(1+\frac{\bar{X}^{2}}{\widehat{\operatorname{var}}(X)}\right) \operatorname{var}(\varepsilon)
\end{aligned}
$$


with $n$ the number of observations, $\widehat{\operatorname{var}}(X)=\sum_{i}\left(X_{i}-\bar{X}\right)^{2} / n$, and $\operatorname{var}(\varepsilon)$ the variance of the regression error (the unexplained excess return). In our context, all models use the same test returns $Y$ (in excess of the risk-free rate). So for a given $\widehat{\operatorname{var}}(Y)$, the regression $R^{2}$ determines the estimate of $\operatorname{var}(\varepsilon)$ in $(1)$. This is the channel that Fama and French (2012) frequently refer to.

For a given error variance, however, the intercept is also estimated imprecisely when the sample variance of the regressor is small relative to its squared mean. For example, if the test returns all have very similar covariances with the market excess return, a zero cross-sectional alpha is more easily accepted.

In the multifactor case, imprecise estimates $\widehat{\alpha}=\bar{Y}-\widehat{\beta}^{\prime} \bar{X}$ can also arise from multicollinearity. Equation (1) generalizes to

$$
\operatorname{var}(\widehat{\alpha} \mid X)=\frac{1}{n}\left(1+\bar{X}^{\prime} \widehat{\operatorname{var}}(X)^{-1} \bar{X}\right) \operatorname{var}(\varepsilon),
$$

with $\widehat{\operatorname{var}}(X)=\sum_{i}\left(X_{i}-\bar{X}\right)\left(X_{i}-\bar{X}\right)^{\prime} / n$. Hence, $\alpha$ will be estimated imprecisely when the factors are highly collinear. Again, the message is that anything that adversely affects the precision of the slope estimates also adversely affects the standard error of the intercept estimate.

\section{Comparative $p$-values}

Consider two competing asset pricing models, Model 1 and Model 2, each with its own set of factors, $X_{1}$ and $X_{2}$. For any asset excess return $Y$, Model $j$ specifies the conditional mean excess return, given information set $I$, as $E(Y \mid I)=\alpha_{j}+\beta_{j}^{\prime} X_{j}$ with $\alpha_{j}=0$ (zero pricing error). Let $\widehat{\alpha}_{j}$ be an approximately unbiased, consistent, and asymptotically normal estimate of $\alpha_{j}$, with consistent standard error estimate $\operatorname{se}\left(\widehat{\alpha}_{j}\right)$. The $\widehat{\alpha}_{j}$ may be OLS, GLS, or GMM estimates, and se $\left(\widehat{\alpha}_{j}\right)$ may be a heteroskedasticity and/or autocorrelation robust estimate. We first define comparative $p$-values for the $t$-test and then for the Wald test.

\subsection{Comparative $p$-values for a single alpha: definition}

Using normal-theory asymptotic approximations, the $p$-value of the null $H_{0}: \alpha_{j}=0$ is

$$
p_{j}=\operatorname{Pr}\left[|Z|>\left|t_{j}\right|\right]=2 \Phi\left(-\left|t_{j}\right|\right),
$$


where $t_{j}=\widehat{\alpha}_{j} / \operatorname{se}\left(\widehat{\alpha}_{j}\right)$ is the $t$-statistic, $Z$ is a standard normal variate, and $\Phi(\cdot)$ is the standard normal distribution function.

Now, if one compares $p_{1}$ with $p_{2}$, any difference in the standard errors $\operatorname{se}\left(\widehat{\alpha}_{1}\right)$ and $\operatorname{se}\left(\widehat{\alpha}_{2}\right)$ is ignored. It may thus occur that, for example, $\left|\widehat{\alpha}_{2}\right|>\left|\widehat{\alpha}_{1}\right|>0$ and $\operatorname{se}\left(\widehat{\alpha}_{2}\right) \gg$ se $\left(\widehat{\alpha}_{1}\right)$, resulting in $p_{1}<p_{2}$. In this case, Model 1 delivers a smaller pricing error, but, according to the $p$-value, Model 2 does better at the zero-alpha test because its alpha estimate is much less precise. The situation may arise, for example, when Model 2 adds a not-so-relevant factor to Model 1 that is highly collinear with the other factors. Obviously, it would be questionable, then, to claim success of Model 2 over Model 1 based on the $p$-value comparison alone. The problematic aspect in this comparison is the unequal precision of the alpha estimates, that is, $\operatorname{se}\left(\widehat{\alpha}_{1}\right) \neq \operatorname{se}\left(\widehat{\alpha}_{2}\right)$. The comparative $p$-values that we now define are intended to correct for this.

Without loss of generality, suppose $\operatorname{se}\left(\widehat{\alpha}_{1}\right)<\operatorname{se}\left(\widehat{\alpha}_{2}\right)$. To equalize the precision of the two alpha estimates, we somehow must raise $\operatorname{se}\left(\widehat{\alpha}_{1}\right)$ to the level of $\operatorname{se}\left(\widehat{\alpha}_{2}\right)$. This requires adding noise to $\widehat{\alpha}_{1}$. Thus, we replace $\widehat{\alpha}_{1}$, as an estimate of $\alpha_{1}$, with the random variable

$$
\widehat{\alpha}_{1}^{*}=\widehat{\alpha}_{1}+\sqrt{\operatorname{se}^{2}\left(\widehat{\alpha}_{2}\right)-\mathrm{se}^{2}\left(\widehat{\alpha}_{1}\right)} W
$$

where $W$ is a standard normal variate independent of $\widehat{\alpha}_{1}$. Clearly, $\widehat{\alpha}_{1}^{*}$ inherits the approximate unbiasedness of $\widehat{\alpha}_{1}$ for $\alpha_{1}$. Furthermore, $\operatorname{se}\left(\widehat{\alpha}_{1}^{*}\right)=\operatorname{se}\left(\widehat{\alpha}_{2}\right)$, so $\widehat{\alpha}_{1}^{*}$ and $\widehat{\alpha}_{2}$ have equal precision, as desired. For given $W$, the $t$-statistic based on $\widehat{\alpha}_{1}^{*}$ is

$$
t_{1}^{*}=\frac{\widehat{\alpha}_{1}^{*}}{\operatorname{se}\left(\widehat{\alpha}_{2}\right)}=a t_{1}+\sqrt{1-a^{2}} W
$$

where $a=\operatorname{se}\left(\widehat{\alpha}_{1}\right) / \operatorname{se}\left(\widehat{\alpha}_{2}\right)$. We define the comparative $p$-values as

$$
\begin{aligned}
p_{1}^{*} & =\operatorname{Pr}\left[|Z|>\left|t_{1}^{*}\right|\right]=2 \operatorname{Pr}\left[Z<-\left|a t_{1}+\sqrt{1-a^{2}} W\right|\right] \\
& =2 \mathrm{E}_{W}\left[\Phi\left(-\left|a t_{1}+\sqrt{1-a^{2}} W\right|\right)\right], \\
p_{2}^{*} & =p_{2},
\end{aligned}
$$

where $\mathrm{E}_{W}$ denotes the expectation with respect to $W$, holding $a$ and $t_{1}$ fixed. Thus, $p_{1}^{*}$ is the expected $p$-value defined by $t_{1}^{*}$, the expectation being taken over the added noise term $W$. Like ordinary $p$-values, $p^{*}$-values are always between 0 and 1 . 


\subsection{Comparative $p$-values for a single alpha: properties and discussion}

It is important to note that our procedure to obtain $p_{1}^{*}$ is not the same as the naive procedure of replacing $\operatorname{se}\left(\widehat{\alpha}_{1}\right)$ with $\operatorname{se}\left(\widehat{\alpha}_{2}\right)$ in $t_{1}$ and computing the $p$-value defined by this modified $t_{1}$. This would give $2 \Phi\left(-\left|a t_{1}\right|\right)$ as a modified $p$-value and, hence, ignore the noise term $W$ required to establish equal precision of the alpha estimates. Intuitively, when a higher standard error is considered in a thought experiment, it makes no sense to assume the estimate of alpha would not be affected, so one also needs to take into account the extent to which the estimated alpha might have been different from its current value.

Figure 1 shows, for a selection of values of $p_{1}$, how $p_{1}$ is transformed into $p_{1}^{*}$ as a function of $\operatorname{se}\left(\widehat{\alpha}_{1}\right) / \operatorname{se}\left(\widehat{\alpha}_{2}\right)$. The curves describe how quickly the statistical evidence against the zero-alpha null fades away as the standard error deteriorates, that is, as we move from right to left along a curve. For example, suppose we originally observe $p_{1}=0.001$ and we consider a scenario under which $\alpha_{1}$ would have been estimated with a standard error that is twice as high as the actual one. This corresponds, for example, to having only one-fourth of the current number of observations (under the same sampling rule). Our expectation of the $p$-value that would have obtained under those circumstances is $p_{1}^{*}=0.20$, the number read from the lower curve $\left(p_{1}=0.001\right)$ at $\operatorname{se}\left(\widehat{\alpha}_{1}\right) / \operatorname{se}\left(\widehat{\alpha}_{2}\right)=0.5$.

Naturally, when $\operatorname{se}\left(\widehat{\alpha}_{1}\right)<\operatorname{se}\left(\widehat{\alpha}_{2}\right)$ and $p_{1}$ is small, $p_{1}^{*}$ exceeds $p_{1}$, as Figure 1 illustrates. (We note that, for sufficiently large values of $p_{1}$, clearly $p_{1}^{*}<p_{1}$, but this can only happen by chance, even if the null holds.) This may appear to be a weakness of comparative $p$-values as we defined them, since, if we were tempted to interpret them as ordinary $p$-values (i.e., measuring evidence against the null), the evidence would be weakened. We reiterate that the primary role of comparative $p$-values lies in their comparability across models, not in their stand-alone capacity as a summary measure to evaluate a given model.

Standard $p$-values (in an approximate normal-theory setting) are defined by the own point estimates and standard errors, that is, Model $j$ 's $p$-value is a function $p_{j}=$ $p\left(\widehat{\alpha}_{j}\right.$, se $\left.\left(\widehat{\alpha}_{j}\right)\right)$. The $p^{*}$-values are defined, in addition, by the standard error of the other model, Model $i$, that is, $p_{j}^{*}=p^{*}\left(\widehat{\alpha}_{j}\right.$, se $\left(\widehat{\alpha}_{j}\right)$, se $\left.\left(\widehat{\alpha}_{i}\right)\right)$. Therefore, a $p^{*}$-value cannot be seen 
Figure 1: Comparative $p$-values

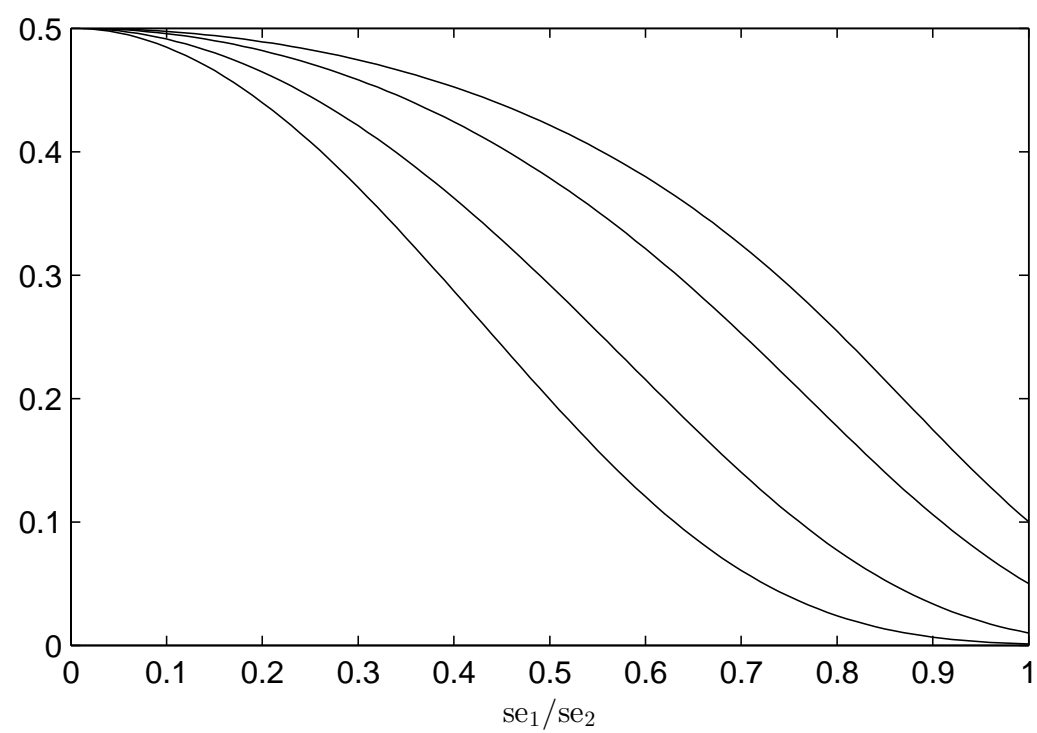

$p_{1}^{*}$ as a function of $\operatorname{se}\left(\widehat{\alpha}_{1}\right) / \operatorname{se}\left(\widehat{\alpha}_{2}\right)$ for $p_{1}=0.1,0.05,0.01,0.001$ (top to bottom).

as a refinement of a stand-alone $p$-value. Moreover, the classic performance concepts of error-in-rejection-probability (under the null) and power (under alternatives) do not apply to $p^{*}$-values, and neither can $p^{*}$-values and $p$-values be compared (or, for that matter, their performances). All that $p^{*}$-values do is recalculate the expected credibility of the null hypothesis of each of the two models in the hypothetical setting where the uncertainty about the models' point estimates is equalized. The more precisely estimated model never becomes more acceptable, but one may, by comparison, become less impressed with the zero-alpha tests of the imprecisely estimated model.

How do the $p^{*}$-values behave as the sample size increases? As $n \rightarrow \infty$, the ratio of standard errors $a=\operatorname{se}\left(\widehat{\alpha}_{1}\right) / \operatorname{se}\left(\widehat{\alpha}_{2}\right)$ converges in probability to a positive constant, $\operatorname{plim} a$. Further, from the definition of the $p^{*}$-values, it is easily seen that, regardless of the value of plim $a$, we have $p_{j} \rightarrow 0$ if and only if $p_{j}^{*} \rightarrow 0$. Hence, in the presence of a pricing error $\left(\alpha_{j} \neq 0\right), p_{j}^{*}$ converges in probability to 0 (since then $\left|t_{j}\right| \rightarrow \infty$ and $p_{j} \rightarrow 0$ ). In the absence of a pricing error $\left(\alpha_{j}=0\right), p_{j}^{*}$ has a non-degenerate limit distribution on $[0,1]$ that depends on plim $a$. This distribution is uniform when plim $a \geq 1$ (since then $p_{j}^{*}=p_{j}$ ), is close to uniform when plim $a$ is less than but close to 1 (since then $p_{j}^{*} \simeq p_{j}$ ), has relatively more mass in the area around 0.5 as plim $a$ gets smaller, as can be read from Figure 1, and becomes degenerate at 0.5 in the $\operatorname{limit} \operatorname{plim} a \rightarrow 0$.

To further clarify the interpretation of the $p^{*}$-values, we ran a small simulation ex- 
Table 1: Simulations

\begin{tabular}{|c|c|c|c|c|c|c|c|c|c|c|}
\hline \multirow[b]{2}{*}{$\alpha_{1}$} & \multirow[b]{2}{*}{$\alpha_{2}$} & \multicolumn{4}{|c|}{$p$-values } & \multicolumn{2}{|c|}{$p^{*}$-values } & \multicolumn{3}{|c|}{ expectations } \\
\hline & & $p_{1}<1 \%$ & $p_{1}<5 \%$ & $p_{2}<1 \%$ & $p_{2}<5 \%$ & $p_{1}^{*}<1 \%$ & $p_{1}^{*}<5 \%$ & $\mathrm{E}\left(p_{1}\right)$ & $\mathrm{E}\left(p_{2}\right)$ & $\mathrm{E}\left(p_{1}^{*}\right)$ \\
\hline \multicolumn{11}{|c|}{$\operatorname{se}\left(\widehat{\alpha}_{1}\right)=0.1, \operatorname{se}\left(\widehat{\alpha}_{2}\right)=0.15, a=2 / 3$} \\
\hline 0 & 0 & 0.010 & 0.050 & 0.010 & 0.050 & 0.000 & 0.000 & 0.500 & 0.500 & 0.500 \\
\hline 0.1 & 0.1 & 0.057 & 0.170 & 0.029 & 0.103 & 0.000 & 0.004 & 0.365 & 0.434 & 0.434 \\
\hline 0.2 & 0.2 & 0.284 & 0.517 & 0.107 & 0.266 & 0.002 & 0.048 & 0.145 & 0.286 & 0.286 \\
\hline 0.4 & 0.4 & 0.923 & 0.979 & 0.536 & 0.760 & 0.207 & 0.632 & 0.005 & 0.058 & 0.058 \\
\hline \multicolumn{11}{|c|}{$\operatorname{se}\left(\widehat{\alpha}_{1}\right)=0.075, \operatorname{se}\left(\widehat{\alpha}_{2}\right)=0.15, a=1 / 2$} \\
\hline 0 & 0 & 0.010 & 0.050 & 0.010 & 0.050 & 0.000 & 0.000 & 0.500 & 0.500 & 0.500 \\
\hline 0.1 & 0.1 & 0.107 & 0.265 & 0.029 & 0.102 & 0.000 & 0.000 & 0.286 & 0.434 & 0.434 \\
\hline 0.2 & 0.2 & 0.536 & 0.759 & 0.107 & 0.267 & 0.000 & 0.006 & 0.058 & 0.286 & 0.286 \\
\hline 0.4 & 0.4 & 0.997 & 1.000 & 0.536 & 0.760 & 0.070 & 0.563 & 0.000 & 0.058 & 0.058 \\
\hline
\end{tabular}

Alpha estimates generated as $\widehat{\alpha}_{j} \sim \mathcal{N}\left(\alpha_{j}, \operatorname{se}\left(\widehat{\alpha}_{j}\right)^{2}\right)$ for fixed $\alpha_{j}$ and $\operatorname{se}\left(\widehat{\alpha}_{j}\right), j=1,2$. Note that $p_{2}^{*}=p_{2}$ since $a<1$.

periment that mimics some key aspects of the application in Section 4 using monthly return data on ten book-to-market test portfolios. In the simulations we leave the DGP that generates the returns unspecified. Instead, in line with the discussion in Section 2, we note that different models $j$ typically yield different pricing errors, $\alpha_{j}$, and different standard errors of the associated estimates, se $\left(\widehat{\alpha}_{j}\right)$. Therefore, we simply take the pricing errors and the standard errors as fixed inputs of the experimental design to generate $\widehat{\alpha}_{j} \sim \mathcal{N}\left(\alpha_{j}, \operatorname{se}\left(\widehat{\alpha}_{j}\right)^{2}\right)$ and subsequently compute $p_{j}$ and $p_{j}^{*}$ for Models $j=1,2$. We set $\alpha_{j}=0,0.1,0.2,0.4$, which corresponds to pricing errors that range from zero to 40 basis points per month, and $\left(\operatorname{se}\left(\widehat{\alpha}_{1}\right), \operatorname{se}\left(\widehat{\alpha}_{2}\right)\right)=(0.1,0.15),(0.75,0.15)$, which corresponds to standard error ratios $a$ of Model 1 to Model 2 equal to $2 / 3$ or $1 / 2$. The simulation results, based on 100,000 Monte Carlo replications for each design point, are given in Table 1. In line with the theory, the $p$-values are uniformly distributed when the pricing errors are zero and tend to zero as the pricing errors increase or the standard errors decrease. Also, for non-zero but equal pricing errors $\alpha_{1}=\alpha_{2} \neq 0$, the distribution of $p_{2}$ stochastically dominates that of $p_{1}$, so Model 2 passes the zero-alpha test more easily than does Model 1. To compensate for the unequal precision of the alpha estimates, low $p_{1}$-values are shifted upward to give $p_{1}^{*}$, which has much less mass in the vicinity of zero. The upward shifting of mass is greater as a gets smaller (recall Figure 1). Nevertheless, when Model 1's pricing error is large $\left(\alpha_{1}=0.4\right), p_{1}^{*}$ still has considerable mass close to zero $(62.3 \%$ of the mass is below 0.05 when $a=2 / 3$, and $56.6 \%$ is below 0.05 when $a=1 / 2)$. Finally, since $p_{1}^{*}$ is constructed as an expected $p$-value under equalization of the precisions of $\widehat{\alpha}_{1}$ and $\widehat{\alpha}_{2}$, the last two columns of the Table 1 are identical. Indeed, 
when $\alpha_{1}=\alpha_{2}$, we have $\mathrm{E}\left(p_{2}\right)=\mathrm{E}\left(p_{2}^{*}\right)=\mathrm{E}\left(p_{1}^{*}\right)$ for every $a$.

Finally, under the additional and strong assumption that the regression errors are i.i.d. normal, a finite-sample comparative $p$-value can be defined. Let $\widehat{\alpha}_{j}$ be the OLS estimate of $\alpha_{j}$, se $\left(\widehat{\alpha}_{j}\right)$ the classic OLS standard error, and $t_{j}=\widehat{\alpha}_{j} / \operatorname{se}\left(\widehat{\alpha}_{j}\right)$. The finitesample $p$-value of $H_{0}$ is

$$
p_{j}=\operatorname{Pr}\left[\left|T_{n-k_{j}-1}\right|>\left|t_{j}\right|\right] \text {, }
$$

where $T_{n-k_{j}-1}$ is a $t$ variate with $n-k_{j}-1$ degrees of freedom and $k_{j}$ is the number of factors of Model $j$. Again, suppose $a=\operatorname{se}\left(\widehat{\alpha}_{1}\right) / \operatorname{se}\left(\widehat{\alpha}_{2}\right)<1$ without loss of generality. Conditioning on $a$ and reasoning as above leads to comparative $p$-values defined as

$$
\begin{aligned}
p_{1}^{*} & =\operatorname{Pr}\left[\left|a T_{n-k_{j}-1}+\sqrt{1-a^{2}} Z\right|>\left|a t_{1}+\sqrt{1-a^{2}} W\right|\right] \\
& =2 \operatorname{Pr}\left[a T_{n-k_{j}-1}+\sqrt{1-a^{2}} Z<-\left|a t_{1}+\sqrt{1-a^{2}} W\right|\right], \\
p_{2}^{*} & =p_{2},
\end{aligned}
$$

where the probability is over the independent random variables $T_{n-k_{j}-1}, Z \sim \mathcal{N}(0,1)$, and $W \sim \mathcal{N}(0,1)$ for fixed $a$ and $t_{1}$. Because $a T_{n-k_{j}-1}+\sqrt{1-a^{2}} Z$ has slightly more mass in the tails than $Z$, the resulting $p_{1}^{*}$ is slightly higher than the asymptotic version of $p_{1}^{*}$ developed above, in the same way that the finite-sample $p$-value is slightly higher than the asymptotic $p$-value. For every $a>0, a T_{n-k_{j}-1}+\sqrt{1-a^{2}} Z$ is standard normal in the limit $n \rightarrow \infty$, so the difference between the finite-sample and the asymptotic version of $p^{*}$ is vanishingly small.

\subsection{Comparative $p$-values for a set of alphas}

We now turn to multiple-alpha tests and comparative $p$-values. For $m$ test assets, let $\widehat{\alpha}_{j}$ be the $m$-vector of the estimated pricing errors of Model $j$, with estimated variance matrix $\widehat{V}_{j}$ (assumed consistent and nonsingular). The Wald statistic of the null $H_{0}$ : $\alpha_{j}=0$ is $\widehat{\alpha}_{j}^{\prime} \widehat{V}_{j}^{-1} \widehat{\alpha}_{j}$, with $p$-value

$$
p_{j}=\operatorname{Pr}\left[\mathcal{X}_{m}^{2}>\widehat{\alpha}_{j}^{\prime} \widehat{V}_{j}^{-1} \widehat{\alpha}_{j}\right]
$$

where $\mathcal{X}_{m}^{2}$ is a $\chi_{m}^{2}$ variate. Diagonalize $\widehat{V}_{1}$ and $\widehat{V}_{2}$ simultaneously as

$$
\widehat{V}_{1}=A \Lambda_{1} A^{\prime}, \quad \widehat{V}_{2}=A \Lambda_{2} A^{\prime}
$$


where $\Lambda_{1}$ and $\Lambda_{2}$ are $m \times m$ diagonal positive definite matrices (see, for example, Magnus and Neudecker 2007, p. 23). Put

$$
Q_{1}=A\left(\Lambda_{2}-\Lambda_{1}\right)_{+} A^{\prime}, \quad Q_{2}=A\left(\Lambda_{1}-\Lambda_{2}\right)_{+} A^{\prime}
$$

where, for a matrix $M$, we denote by $M_{+}$the matrix with elements $\left(M_{+}\right)_{i j}=\max \left(M_{i j}, 0\right)$. Let

$$
W_{1} \sim \mathcal{N}\left(0, Q_{1}\right), \quad W_{2} \sim \mathcal{N}\left(0, Q_{2}\right),
$$

with $W_{j}$ independent of $\widehat{\alpha}_{j}$. Define the precision-equalized estimates

$$
\widehat{\alpha}_{1}^{*}=\widehat{\alpha}_{1}+W_{1}, \quad \widehat{\alpha}_{2}^{*}=\widehat{\alpha}_{2}+W_{2},
$$

noting that

$$
\operatorname{var}\left(\widehat{\alpha}_{1}^{*}\right)=\widehat{V}_{1}+Q_{1}=A \max \left(\Lambda_{1}, \Lambda_{2}\right) A^{\prime}=\widehat{V}_{2}+Q_{2}=\operatorname{var}\left(\widehat{\alpha}_{2}^{*}\right)
$$

where $\max \left(\Lambda_{1}, \Lambda_{2}\right)$ is the matrix of element-wise maxima of $\Lambda_{1}$ and $\Lambda_{2}$. The matrices $Q_{1}$ and $Q_{2}$ are the smallest positive semi-definite matrices such that $\widehat{V}_{1}+Q_{1}=\widehat{V}_{2}+Q_{2}$. The comparative $p$-values are defined, for $j=1,2$, as

$$
\begin{aligned}
p_{j}^{*} & =\operatorname{Pr}\left[\mathcal{X}_{m}^{2}>\widehat{\alpha}_{j}^{* \prime}\left(\widehat{V}_{j}+Q_{j}\right)^{-1} \widehat{\alpha}_{j}^{*}\right] \\
& =\operatorname{Pr}\left[\mathcal{X}_{m}^{2}>\left(\widehat{\alpha}_{j}+W_{j}\right)^{\prime}\left(\widehat{V}_{j}+Q_{j}\right)^{-1}\left(\widehat{\alpha}_{j}+W_{j}\right)\right],
\end{aligned}
$$

where the probability is taken over the independent random variables $\mathcal{X}_{m}^{2}$ and $W_{j}$, holding $\widehat{\alpha}_{j}, \widehat{V}_{j}$, and $Q_{j}$ fixed.

\section{Application}

Liu (2006) proposes a liquidity-augmented CAPM and compares it with the CAPM and the Fama and French (1993) three-factor model. Commenting on the time-series regressions, tested on ten book-to-market portfolios using monthly data from January 1964 to December 2003, Liu (2006, p. 664 and 666) notes that "In contrast to the [... ] Fama and French three-factor model, the two-factor model explains the returns of all B/M decile portfolios". As we see in Table 2, the number of large $t$-statistics (exceeding 2 in absolute value) does, indeed, drop from four to zero. However, the last line of the table also tells us that, for the ten regressions, the standard errors of the estimated alphas in the liquidity-augmented CAPM are between 1.846 and 2.566 times as large as 
Table 2: Fama-French three-factor model and liquidity-augmented CAPM

\begin{tabular}{|c|c|c|c|c|c|c|c|c|c|c|}
\hline $\mathrm{B} / \mathrm{M}$ decile & 1 & 2 & 3 & 4 & 5 & 6 & 7 & 8 & 9 & 10 \\
\hline & \multicolumn{10}{|c|}{ Fama-French three-factor model } \\
\hline$\widehat{\alpha}(\%)$ & -0.308 & -0.150 & -0.088 & -0.042 & -0.022 & 0.072 & 0.070 & 0.135 & 0.212 & 0.210 \\
\hline $\operatorname{se}(\widehat{\alpha})$ & 0.086 & 0.061 & 0.056 & 0.055 & 0.057 & 0.060 & 0.058 & 0.064 & 0.076 & 0.112 \\
\hline$t$-statistic & -3.565 & -2.467 & -1.588 & -0.762 & -0.394 & 1.185 & 1.214 & 2.119 & 2.770 & 1.871 \\
\hline$p$ & 0.000 & 0.014 & 0.112 & 0.446 & 0.693 & 0.236 & 0.225 & 0.034 & 0.006 & 0.061 \\
\hline$p^{*}$ & 0.171 & 0.367 & 0.460 & 0.511 & 0.524 & 0.485 & 0.484 & 0.391 & 0.295 & 0.370 \\
\hline \multirow[t]{2}{*}{ Wald test } & \multicolumn{10}{|c|}{ statistic $=36.14, p=7.97 \times 10^{-5}, p^{*}=0.0128$} \\
\hline & \multicolumn{10}{|c|}{ liquidity-augmented CAPM } \\
\hline$\widehat{\alpha}(\%)$ & -0.111 & 0.019 & 0.046 & 0.065 & 0.074 & 0.150 & 0.128 & 0.154 & 0.186 & 0.118 \\
\hline $\operatorname{se}(\widehat{\alpha})$ & 0.174 & 0.148 & 0.143 & 0.137 & 0.139 & 0.142 & 0.139 & 0.146 & 0.165 & 0.207 \\
\hline$t$-statistic & -0.638 & 0.129 & 0.319 & 0.472 & 0.535 & 1.056 & 0.922 & 1.053 & 1.128 & 0.571 \\
\hline$p=p^{*}$ & 0.524 & 0.897 & 0.750 & 0.637 & 0.593 & 0.291 & 0.357 & 0.293 & 0.260 & 0.568 \\
\hline \multirow[t]{3}{*}{ Wald test } & \multicolumn{10}{|c|}{ statistic $=12.57, p=0.249, p^{*}=0.247$} \\
\hline & \multicolumn{10}{|c|}{ ratio of $\operatorname{se}(\widehat{\alpha})$ of liquidity-augmented CAPM to three-factor model } \\
\hline & 2.012 & 2.433 & 2.566 & 2.493 & 2.453 & 2.351 & 2.399 & 2.293 & 2.162 & 1.846 \\
\hline
\end{tabular}

Source: the $\widehat{\alpha}$ 's and $t$-statistics are from Liu (2006, Table 9, Panel B); the rest is inferred from the same underlying data.

the corresponding standard errors in the Fama-French model. ${ }^{2}$ This raises the question whether the prima facie success of the liquidity-augmented CAPM, as diagnosed via the zero-alpha tests, might primarily be the result of imprecise estimates (Fama and French's "too little power" problem).

From the asymptotic $p$-values in Table 2, we see that the Fama-French three-factor model misprices the two smallest-cap decile portfolios and the three biggest-cap ones, against none for the liquidity-augmented CAPM, but that is, to at least some extent, due to the latter's larger standard errors. In terms of absolute pricing errors, the liquidityaugmented CAPM does better for the small-cap decile portfolios but worse for the midcaps. To see whether the bigger standard errors are the main explanation, we compute the asymptotic comparative $p$-values, $p^{*}$. These $p^{*}$ s tell us that none of the Fama-French three-factor model's pricing errors would have been deemed significant if the three-factor model's estimates had been as imprecise as those of the liquidity-augmented CAPM. The conclusion, then, is not that the three-factor model fares better at the zero-alpha tests

\footnotetext{
${ }^{2}$ Proxies for liquidity inevitably resemble size and, to a lesser extent, "value", so a multicollinearity issue may arise when a liquidity variable is added to a size-augmented CAPM. When size is omitted, the intercept of a liquidity-augmented CAPM is still estimated more noisily if the $R^{2}$ is not as good as in the competing model and especially so if market liquidity also falls when markets fall (i.e., a multicollinearity problem may arise in addition to a lower $R^{2}$ ).
} 
than the unmodified $p$-values suggest - the pricing errors are statistically significant, after all-but that the apparent relative success of the liquidity-based CAPM is largely due to imprecisely estimated alphas.

Table 2 also reports the Wald test for the joint zero-alpha hypothesis on all ten $\mathrm{B} / \mathrm{M}$ decile portfolios. The Wald statistic is $\widehat{\alpha}^{\prime} \widehat{V}^{-1} \widehat{\alpha}$, where $\widehat{V}=\frac{1}{n}\left(1+\bar{X}^{\prime} \widehat{\operatorname{var}}(X)^{-1} \bar{X}\right) \widehat{\Sigma}$ and $\widehat{\Sigma}$ is the variance matrix of the multivariate regression residuals. The joint null of zero pricing errors is overwhelmingly rejected in the Fama-French three-factor model while it is accepted in the liquidity-augmented CAPM. Here, again, the strong evidence against the three-factor model is partly due to the higher precision of the estimated alphas. The difference between the $\widehat{V}^{\prime}$ 's in the liquidity-augmented CAPM and the three-factor

model, $\widehat{V}_{\mathrm{LIQ}}-\widehat{V}_{\mathrm{FF} 3 \mathrm{~F}}$, is almost positive definite: there is only one negative eigenvalue and it is very close to zero. As a result, the $p^{*}$-value for the liquidity-augmented CAPM is virtually unchanged relative to the $p$-value, while, for the three-factor model, it is orders of magnitude greater than the $p$-value. Nevertheless, in the three-factor model, $p^{*}$ stays well below the $5 \%$ level, so the relatively easy acceptance of the liquidity-augmented CAPM in light of the $p$-values is only partly due to $\widehat{V}_{\text {FF3F }}$ being less than $\widehat{V}_{\text {LIQ }}$.

\section{Concluding remarks}

Comparing the statistical significance of pricing errors across asset pricing models is problematic since it favors more noisily estimated models, as is widely recognized. In the GMM setting, this is one motivation for the Hansen-Jagannathan distance, which uses a fixed weight matrix in the GMM objective function instead of the model-specific optimal weight matrix. Different models then use the same weight and, therefore, their implied pricing errors are being evaluated on a more equal footing. The $p$-value associated with the Hansen-Jagannathan distance remains a genuine $p$-value in the sense of being a statistical measure of evidence against model misspecification. But, as with any $p$-value, it inevitably reflects the degree of deviation from the null and the underlying statistical precision of the model estimates. Among models with a nonzero but equal degree of mispricing (in the population sense), imprecisely estimated models will still be favored. The comparative $p$-values suggested in this paper are a way to fully eliminate the effect of unequal precisions on $p$-values.

We repeat that the usual interpretation of $p$-values is lost, so they should not be 
misinterpreted. They do not indicate how significant an alpha estimate is but shed light on how relatively easy or difficult it is for a model to pass a zero-alpha test in comparison with another model. In our application, for instance, we do not at all claim that the three-factor model's pricing errors are statistically insignificant, nor do we claim that liquidity is an irrelevant factor; rather, we note that, if the Fama-French pricing errors had been estimated as imprecisely as those in the liquidity-augmented CAPM, we would have worried much less about them. This conclusion qualifies the comparison between the models only.

Acknowledgments. We thank two referees for helpful comments and Weimin Liu for generously sharing his data with us.

\section{References}

Asness, Clifford S, Tobias J Moskowitz, and Lasse Heje Pedersen. 2013. "Value and momentum everywhere." The Journal of Finance, 68(3): 929-985.

Banz, Rolf W. 1981. "The relationship between return and market value of common stocks." Journal of Financial Economics, 9(1): 3-18.

Basu, Sanjoy. 1977. "Investment performance of common stocks in relation to their price-earnings ratios: A test of the efficient market hypothesis." The journal of Finance, 32(3): 663-682.

Beaulieu, Marie-Claude, Jean-Marie Dufour, and Lynda Khalaf. 2013. "Identification-robust estimation and testing of the zero-beta CAPM." The Review of Economic Studies, 80(3): 892-924.

Black, Fischer, Michael C Jensen, and Myron S Scholes. 1972. "The capital asset pricing model: Some empirical tests." Studies in the Theory of Capital Markets, , ed. Michael C. Jensen, 79-121. Praeger Publishers.

Carhart, Mark M. 1997. "On persistence in mutual fund performance." The Journal of Finance, 52(1): 57-82.

Cochrane, John H. 2005. Asset pricing. Princeton University Press. 
Fama, Eugene F, and James D MacBeth. 1973. "Risk, return, and equilibrium: Empirical tests." The Journal of Political Economy, 81(3): 607-636.

Fama, Eugene F, and Kenneth R French. 1992. "The cross-section of expected stock returns." The Journal of Finance, 47(2): 427-465.

Fama, Eugene F, and Kenneth R French. 1993. "Common risk factors in the returns on stocks and bonds." Journal of Financial Economics, 33(1): 3-56.

Fama, Eugene F, and Kenneth R French. 1996. "Multifactor explanations of asset pricing anomalies." The Journal of Finance, 51(1): 55-84.

Fama, Eugene F, and Kenneth R French. 2012. "Size, value, and momentum in international stock returns." Journal of Financial Economics, 105(3): 457-472.

Fama, Eugene F, and Kenneth R French. 2015. "A five-factor asset pricing model." Journal of Financial Economics, 116(1): 1-22.

Gibbons, Michael R. 1982. "Multivariate tests of financial models: A new approach." Journal of Financial Economics, 10(1): 3-27.

Gibbons, Michael R, Stephen A Ross, and Jay Shanken. 1989. "A test of the efficiency of a given portfolio." Econometrica, 1121-1152.

Hansen, Lars Peter. 1982. "Large sample properties of generalized method of moments estimators." Econometrica, 50(4): 1029-1054.

Hansen, Lars Peter, and Ravi Jagannathan. 1997. "Assessing specification errors in stochastic discount factor models." The Journal of Finance, 52(2): 557-590.

Jagannathan, Ravi, and Zhenyu Wang. 1996. "The conditional CAPM and the cross-section of expected returns." The Journal of Finance, 51(1): 3-53.

Jegadeesh, Narasimhan, and Sheridan Titman. 1993. "Returns to buying winners and selling losers: Implications for stock market efficiency." The Journal of Finance, 48(1): 65-91.

Jensen, Michael C. 1968. "The performance of mutual funds in the period 1945-1964." The Journal of Finance, 23(2): 389-416. 
Jobson, John D, and Bob Korkie. 1982. "Potential performance and tests of portfolio efficiency." Journal of Financial Economics, 10(4): 433-466.

Kandel, Shmuel, and Robert F Stambaugh. 1989. "A mean-variance framework for tests of asset pricing models." The Review of Financial Studies, 2(2): 125-156.

Lewellen, Jonathan, Stefan Nagel, and Jay Shanken. 2010. "A skeptical appraisal of asset pricing tests." Journal of Financial Economics, 96(2): 175-194.

Liu, Weimin. 2006. "A liquidity-augmented capital asset pricing model." Journal of Financial Economics, 82(3): 631-671.

MacKinlay, A Craig, and Matthew P Richardson. 1991. "Using Generalized Method of Moments to Test Mean-Variance Efficiency." The Journal of Finance, 46(2): $511-527$.

Pastor, Lubos, and Robert F. Stambaugh. 2003. "Liquidity Risk and Expected Stock Returns." Journal of Political Economy, 111(3): 642-685.

Ray, Surajit, N Eugene Savin, and Ashish Tiwari. 2009. "Testing the CAPM revisited." Journal of Empirical Finance, 16(5): 721-733.

Shanken, Jay. 1992. "On the estimation of beta-pricing models." Review of Financial studies, 5(1): 1-55.

Stambaugh, Robert F. 1982. "On the exclusion of assets from tests of the twoparameter model: A sensitivity analysis." Journal of Financial Economics, 10(3): 237268. 